Bull. Austral. Math. Soc.

20D10, 20D04

VOL. 38 (1988) [157-158]

\title{
COMPUTATIONAL APPROACHES TO THE THEORY OF FINITE SOLUBLE GROUPS
}

\section{Stephen Peter Glasby}

This thesis is concerned with algorithmic solutions to problems in the theory of finite soluble groups. Such groups may be defined as permutation groups, matrix groups or finitely presented groups. The algorithms presented here are designed for soluble groups which are defined by power-commutator presentations [2]. These presentations are obtained from a normal series $G=N_{1} \triangleright \ldots \triangleright N_{r+1}=\langle 1\rangle$ with elementary abelian quotients. Most of the algorithms use recursion on $r$, where the passage from $G / N_{i}$ to $G / N_{i+1}$ involves a calculation in linear algebra.

Power-commutator presentations of certain nilpotent quotients of finite presentations may be efficiently calculated by using an algorithm called the nilpotent quotient algorithm [5]. In the absence of an efficient soluble quotient algorithm, there is a need for convenient tools for constructing power-commutator presentations. The classical theoretical methods of constructing soluble groups using split extenions, central extensions, cyclic extensions, wreath products, subgroups and quotient groups, have been employed by the author to construct power-commutator presentations [4].

The major problems addressed in this thesis involve the construction of normalisers, intersections and centralisers. All of these problems are at least as difficult as the problem of constructing discrete logarithms [1], and are unlikely to have polynomial solutions. Fortunately, however, the author has found polynomial solutions to many theoretically important subcases of these problems.

The author presents an algorithm which solves the problem of conjugacy of Hall $\pi$-subgroups. This algorithm is fundamental to algorithms for constructing a Hall $\pi$ subgroup, and the normaliser of a Hall $\pi$-subgroup. The latter algorithm has important applications to the construction of Carter subgroups and system normalisers [2].

Given subgroups $H$ and $K$ of a soluble group $G$, the difficulty of computing $H \cap K$ depends on the relationship between $H$ and $K$ and the normal subroups of $G$. For example, if $K$ is normal (or subnormal), then $H \cap K^{-}$may be computed using an algorithm which is reminiscent of one for intersecting subspaces of vector space. More generally, the problem of calculating $H \cap K$ modulo $N_{i+1}$ from $H \cap K$ modulo

Received 19 March, 1987. Thesis submitted to The University of Sydney, February 1987. Degree approved February 1988. Supervisor Dr. J.J. Cannon

Copyright Clearance Centre, Inc. Serial-fee code: 0004-9729/88 \$A2.00+0.00. 
$N_{i}$, depends on the relationship between the subgroups $H$ and $K$, and the section $N_{i} / N_{i+1}$. If $H$ or $K$ covers $N_{i} / N_{i+1}$, then the covering algorithm may be used to construct $H \cap K$ modulo $N_{i+1}$ from $H \cap K$ modulo $N_{i}$. The avoidance algorithm deals with the case when $N_{i} / N_{i+1}$ lies in the centre of $G / N_{i+1}$ and both $H$ and $K$ avoid $N_{i} / N_{i+1}$.

The covering and avoidance algorithms may be used to intersect arbitrary subgroups of a nilpotent group. A more impressive application of the covering algorithm is to the construction of $H \cap K$ when the indices $|G: H|$ and $|G: K|$ are coprime. Consequently, the covering algorithm may be used to construct Sylow bases, system normalisers and certain complements [3].

Although the problem of constructing arbitrary centralisers is a complicated one, certain coprime centralisers may be constructed using the rational canonical form and field extensions. These centralisers are constructed with an algorithm called the coprime centraliser algorithm which may be used to construct characteristic subgroups such as $0_{\pi}(G), \operatorname{Fit}(G), \operatorname{Frat}(G)$ and $Z(G)$. The algorithm for constructing the Frattini subgroup employs theoretical results which avoid the computation of maximal subgroups. Finally, a theoretical result due to Baer is used in conjunction with the algorithm for constructing the normaliser of a Hall $\pi$-subgroup to produce an algorithm for testing whether a group is supersoluble.

\section{REFERENCES}

[1] D. Coppersmith, 'Evaluating logarithms in $G F\left(2^{n}\right)$ ', in Proc. 16th Annual ACM Symp. on the Theory of Computing: Association for Computing Machinery, pp. 201-207, 1984.

[2] S.P. Glesby, 'Constructing normalisers in finite soluble groups', J. Symbolic Computation (1988) (to apperr).

[3] S.P. Glasby, 'Intersecting subgroups of a finite soluble group', J. Symbolic Computation (1988) (to appear).

[4] S.P. Glasby, 'Constructing finite soluble groups', Cayley Bulletin, University of Sydney 3 (1987), 106-112.

[5] G. Havas and M.F. Newman, 'Applications of computers to questions like those of Burnside': Burnside Groups, Proc. Conf. Bielefeld, Germany. Lecture Notes in Mathematic 806, pp. 211-230. (Springer-Verlag, Berlin).

Department of Pure Mathematics

The University of Sydney

New South Wales, 2006

Australia 